\title{
Resolution of Chronic Immune Thrombocytopenia Purpura after Autologous Hematopoietic Stem Cell Transplantation for Diffuse Large B-Cell Lymphoma
}

\author{
Prashanth A. Kumar ${ }^{a, b}$, Ali Wazira, b, Jeffrey J. Pu ${ }^{a, c}$
}

\begin{abstract}
Immune thrombocytopenic purpura (ITP) is a hematological disorder characterized by immune-mediated destruction of platelets that could be triggered by a number of causes. ITPs are usually treated with steroid, immunomodulators or immunosuppressors, and intravenous immunoglobulin therapy though refractory/relapsed status frequently occurs. It was suggested that autologous hematopoietic stem cell transplant (HSCT) after high-dose chemotherapy conditioning might improve ITP patients' peripheral blood platelet counts via reorganizing disrupted immune balance in the hematopoietic and hematologic systems. In this case report, we describe how a patient, who suffered from both severe thrombocytopenia due to chronic ITP and refractory/relapsed diffuse large B-cell lymphoma (DLBCL), was managed to successfully receive autologous HSCT using carmustine, etoposide, cytarabine and melphalan (BEAM) conditioning regimens and how his chronic ITP was eventually cured after receiving autologous HSCT. This is the first clinical case in the world demonstrating that high-dose BEAM chemotherapy conditioned autologous HSCT could cure chronic ITP while successfully managing refractory/relapse DLBCL. The clinical hematology professionals and the patients will benefit from our experience in managing severe thrombocytopenia while conducting high-dose chemotherapy conditioning and autologous HSCT for DLBCL.
\end{abstract}

Keywords: Immune thrombocytopenic purpura; Diffuse large B-cell lymphoma; BEAM conditioning regimens; Hematopoietic stem cell transplantation

\section{Introduction}

Immune thrombocytopenic purpura (ITP) is a hematologi-

Manuscript submitted October 13, 2020, accepted November 3, 2020

Published online November 18, 2020

${ }^{a}$ Department of Medicine, State University of New York Upstate Medical University, Syracuse, NY, USA

${ }^{b}$ The authors equally contributed to this manuscript.

${ }^{\mathrm{c}}$ Corresponding Author: Jeffrey J. Pu, Department of Medicine, State University of New York Upstate Medical University, Suite 331, CWB, 750 E. Adams Street, Syracuse, NY 13210, USA. Email: jeffreypu@gmail.com

doi: https://doi.org/10.14740/jmc3607 cal disorder characterized by immune-mediated destruction of circulating platelets. ITP could be triggered by a number of causes such as inherited diseases, infections, autoimmune conditions and malignancies [1]. It was reported that lymphoma could associate with ITP and successful treatment of lymphoma might resolve ITP [2]. We herein, first in the world, report a unique clinical case and our experience in curing refractory ITP on a patient who received autologous hematopoietic stem cell transplantation (HSCT) using carmustine, etoposide, cytarabine and melphalan (BEAM) conditioning regimens for refractory/relapsed diffuse large B-cell lymphoma (DLBCL).

\section{Case Report}

\section{Investigations}

The patient was a 64-year-old man with a previous medical history of stage 4 DLBCL diagnosed in 2013. His lymphoma was successfully treated with six cycles of rituximab, cyclophosphamide, doxorubicin and vincristine (RCHOP) and he was in remission for 2 years. In 2015, this patient developed significant thrombocytopenia with lowest platelet count of 2 $\times 10^{3} / \mu \mathrm{L}$. He was subsequently diagnosed as having an ITP. His thrombocytopenia did not respond well to both rituximab and steroid managements, but weekly romiplostim (2 $\mu \mathrm{g} / \mathrm{kg}$ ) successfully maintained his platelet above $30 \times 10^{3} /$ $\mu \mathrm{L}$.

\section{Diagnosis}

In 2018 , this patient rapidly developed peripheral lymphadenopathy. A core biopsy of right iliac lymph node confirmed a relapsed DLBCL. Immunohistochemistry showed enhanced expression of C-MYC, BCL-2 and BCL-6, but a subsequent fluorescence in situ hybridization (FISH) ruled out double or triple hit lymphoma. A bone marrow biopsy did not demonstrate any bone marrow involvement. Positron emission tomography/computed tomography (PET/CT) scan showed multifocal progression of soft tissue nodules, with metabolically active lymphadenopathy, especially in the right iliac lymph nodes chain. 

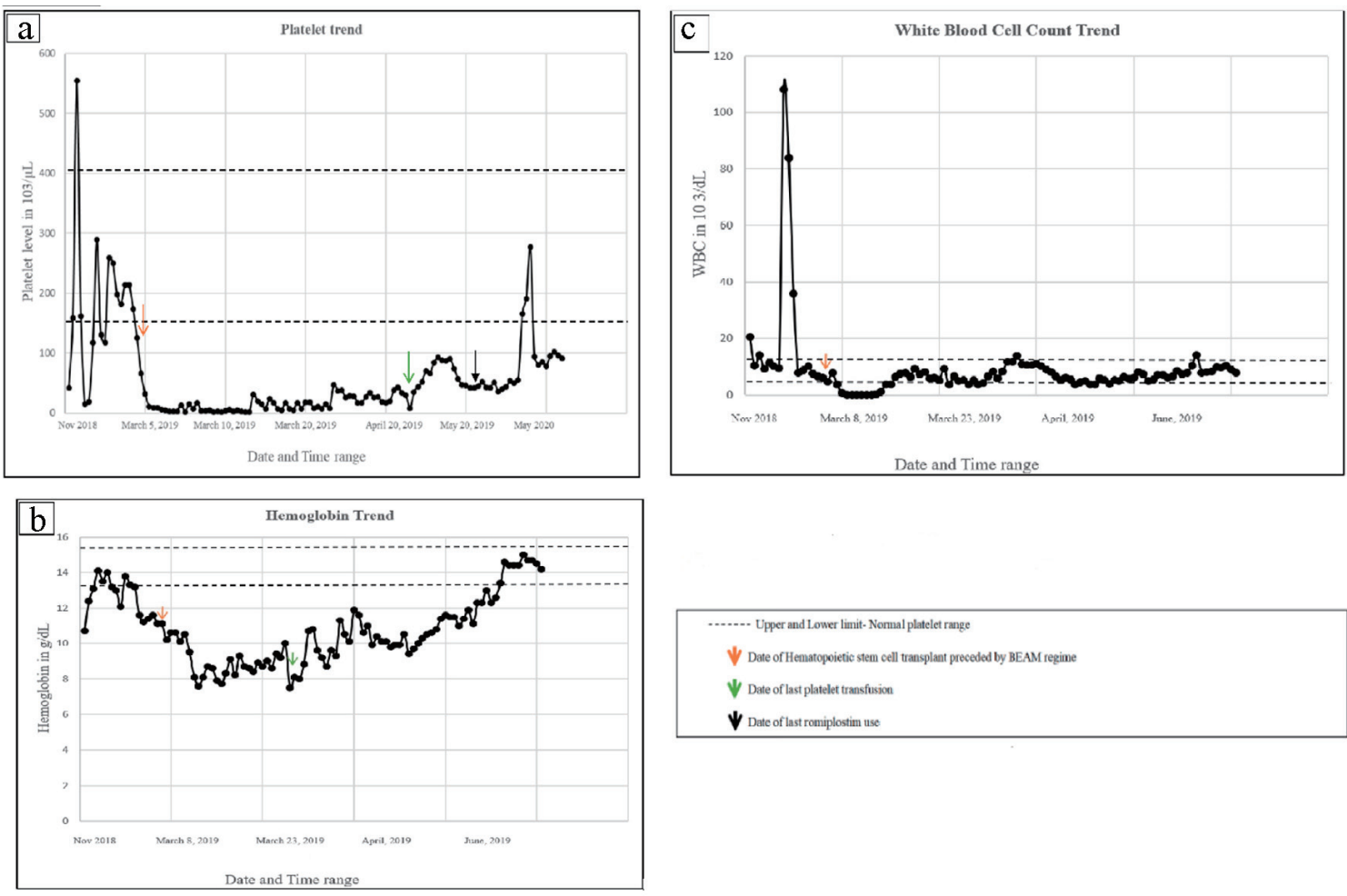

Figure 1. The trends of platelets, hemoglobin and white blood cells over time. (a) Patient received his last platelet transfusion 42 days post HSCT and last romiplostim treatment 75 days post HSCT. His ITP has been in complete remission for 498 days as per electronic medical records and is continuing in remission at current time. (b) Trend of hemoglobin over time in relation to HSCT and last platelet transfusion. (c) Trend of white blood cell count in relation to HSCT. HSCT: hematopoietic stem cell transplant; ITP: immune thrombocytopenic purpura.

\section{Treatment}

He then received two cycles of rituximab, ifosfamide, carboplatin and etoposide (RICE) combination chemotherapy. Post-chemotherapy PET/CT scan showed a morphological complete remission and patient was subsequently referred for autologous HSCT consultation.

Throughout the course of DLBCL treatment, he continuously presented with thrombocytopenia and was managed with romiplostim. He subsequently underwent an autologous HSCT with BEAM conditioning regimen on March 5, 2019. During high-dose BEAM combination chemotherapy conditioning period and first 3 weeks post-transplant, patient received daily twice platelet transfusion to maintain platelet counts $>15 \times$ $10^{3} / \mu \mathrm{L}$, romiplostim $10 \mu \mathrm{g} / \mathrm{kg}$ subcutaneous (SQ) weekly, intravenous immunoglobulin (IVIG) weekly and steroids support.

\section{Follow-up and outcomes}

His post-transplant course was complicated by systemic infection and several episodes of paroxysmal A fib with rapid ventricular response (RVR), which was controlled with Cardizem $120 \mathrm{mg}$ XR oral daily, flecainide $100 \mathrm{mg}$ oral twice daily. Interestingly, his platelet counts recovered nicely, having not required platelet transfusion since April 16, 2019 and not required romiplostim treatment since May 19, 2019 (Fig. 1). PET/CT scans 1 year post transplant showed a complete remission of DLBCL (Fig. 2).

\section{Discussion}

The prevalence of ITP in patient's non-Hodgkin's lymphoma (NHL) is around $0.76 \%$ [3]. Anecdotal evidence suggests that platelet level may predict outcomes in DLBCL [4]. The international prognostic index (IPI) and hemoglobin platelet (HP) index have been analyzed, but the level of evidence remains to be weak and controversial $[4,5]$. It is believed that lymphoma tissues produce autoantibodies against platelets that may incite ITP [2]. Elevated IgG and IgM antiplatelets have been demonstrated in both in vitro and in vivo models supporting this hypothesis [6]. Around 10 cases of ITP associated with DLBCL have been reported in literature, highlighting the uniqueness of this presentation [2].

DLBCL is an aggressive form of NHL in which, complete remission can be attained with optimal therapy. In the present era rituximab and anthracycline-based chemotherapeutic regimens such as RCHOP have remained the standard of care [7]. RCHOP fails in $30-50 \%$ of the cases of DLBCL. Refractory disease occurs in $20 \%$ of the cases, whereas around 

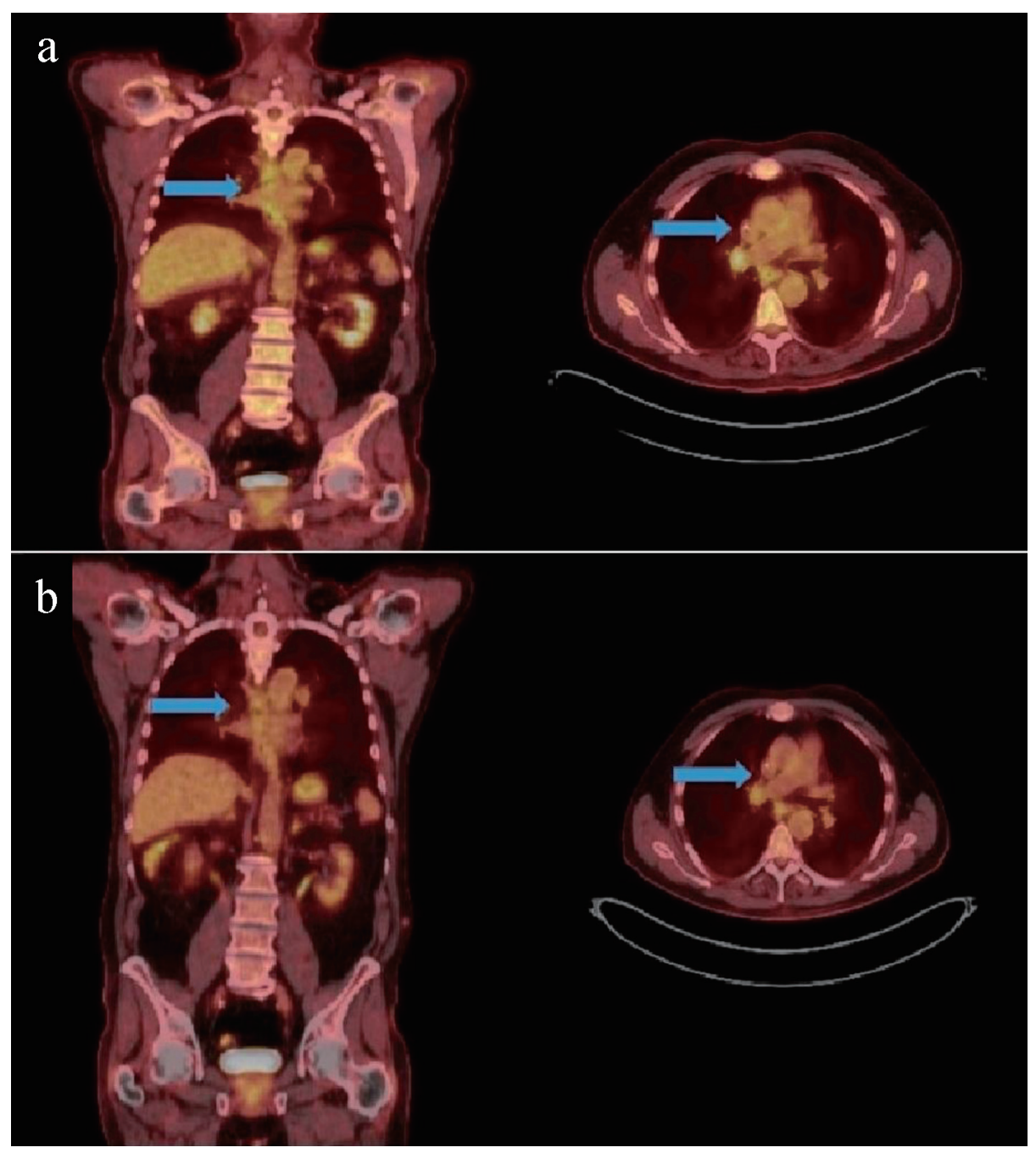

Figure 2. PET/CT monitoring shows that patient's DLBCL is still in remission 1 year after autologous HSCT. (a) PET/CT before HSCT. Inferior right hilar lymph node with SUV max of 5.5 (arrow). (b) PET/CT 1 year after HSCT. Continued decline in the metabolic activity within the inferior right hilar lymph node with SUV max of 3.9. No new metabolic activity was observed in whole body. $\mathrm{PET/CT}$ : positron emission tomography/computed tomography; DLBCL: diffuse large B-cell lymphoma; HSCT: hematopoietic stem cell transplant; SUV: standardized uptake value.

$30 \%$ will have a relapse even after complete remission (CR) [8]. For these patients, salvage cytotoxic chemotherapy with regimens like RICE followed by autologous HSCT results in long-term survival benefits in around $50 \%$ of the patients. For patients who do not respond or have a second relapse allogenic HSCT and emerging novel therapies such as chimeric antigen receptor (CAR) T-cell therapy, antibody-drug conjugates (ADCs) and bispecific T-cell engagers (BiTEs) have a role [9].

It was observed that people with NHL could increase the risk of ITP incidence, and successful treatment of the lymphoma also could improve platelet counts. In a series of 10 cases described by Ono et al, six went into remission after treatment for DLBCL with chemotherapy, three of these patients however required splenectomy [2].

HSCT decreases the circulating pool of auto-reactive $T$ and $\mathrm{B}$ cells, effectively resetting the immune system. Thymic removal of self-reactive cells, promotion of self-tolerance and cytotoxic effect of the ablative priming regimen are a few of the proposed mechanisms by which stem cell transplant may provide benefit in refractory ITP [10]. A National Institutes of Health (NIH) phase II study on nine ITP patients and five Evans syndrome patients showed that autologous stem cell transplant had a response rate of $57 \%$ [10]. Another study analyzed 36 patients who either received autologous (27) or allogenic (nine) stem cell transplant for various cytopenias. Four out of 12 ITP patients, who received autologous stem cell transplant, experienced a sustained platelet count response. A subsequent analysis, involving 52 patients with ITP and Evans syndrome, showed an overall survival of $61 \%$. Recurrence-free survival was similar between autologous and allogenic transplant. The inadequate power and small sample size are the main limitations of these trials [11]. The case we present here is the first case showing that autologous stem cell transplant with BEAM 
conditioning for DLBCL could resolve coexisting ITP as well.

\section{Learning points}

The literatures indicated that $0.76 \%$ lymphoma patients could be complicated with chronic ITP [3,4]. ITP-caused severe thrombocytopenia is a huge obstacle for patients who expect to receive HSCT, especially using high-dose multiple chemotherapy regimens combination (e.g. BEAM) as conditioning agent. This case report described our experience in using novel multiple agent combination treatment protocol to manage severe thrombocytopenia during high-dose conditioning regimens chemotherapy period and early post-HSCT period. This case report also first in the world demonstrated that highdose BEAM chemotherapy regimens conditioned autologous HSCT could cure chronic/refractory ITP that was associated with DLBCL.

\section{Acknowledgments}

The authors would like to thank this patient for agreeing to share his experience in managing those complicated and critical medical conditions.

\section{Financial Disclosure}

This study was supported by: AA\&MDSIF research grant to JJP (146818), American Cancer Society grant to JJP (124171IRG-13-043-02), NIDA/FDA research grant to JJP (P50 DA036107), JTTai\&Co Foundation Cancer Research Grant to JJP, Paige's Butterfly cancer research grant, and A SUNY Upstate Medical University research grant to JJP.

\section{Conflict of Interest}

The authors declare that they have no conflict of interest to disclose.

\section{Informed Consent}

Informed consent was obtained from the individual participant included in this study.

\section{Author Contributions}

JJP initiated the research idea and concept. JJP and AW treated this patient. JJP, PK and AW designed this study and wrote this manuscript.

\section{Data Availability}

Any inquiries regarding supporting data availability of this study should be directed to the corresponding author.

\section{References}

1. Zufferey A, Kapur R, Semple JW. Pathogenesis and therapeutic mechanisms in immune thrombocytopenia (ITP). J Clin Med. 2017;6(2):16.

2. Ono K, Onishi Y, Kobayashi M, Ichikawa S, Hatta S, Watanabe S, Okitsu Y, et al. Successful treatment of aggressive mature B-cell lymphoma mimicking immune thrombocytopenic purpura. Intern Med. 2018;57(17):2573-2579.

3. Hauswirth AW, Skrabs C, Schutzinger C, Raderer M, Chott A, Valent P, Lechner K, et al. Autoimmune thrombocytopenia in non-Hodgkin's lymphomas. Haematologica. 2008;93(3):447-450.

4. Chen LP, Lin SJ, Yu MS. Prognostic value of platelet count in diffuse large B-cell lymphoma. Clin Lymphoma Myeloma Leuk. 2012;12(1):32-37.

5. Nakayama S, Matsuda M, Adachi T, Sueda S, Ohashi Y, Awaji S, Hashimoto S, et al. Novel prognostic index based on hemoglobin level and platelet count for diffuse large B-cell lymphoma, not otherwise specified in the RCHOP era. Platelets. 2019;30(5):637-645.

6. Visco C, Rodeghiero F. Immune thrombocytopenia in lymphoproliferative disorders. Hematol Oncol Clin North Am. 2009;23(6):1261-1274.

7. Wong Doo N, White VM, Martin K, Bassett JK, Prince HM, Harrison SJ, Jefford M, et al. The use of optimal treatment for DLBCL is improving in all age groups and is a key factor in overall survival, but non-clinical factors influence treatment. Cancers (Basel). 2019;11(7):928.

8. Lekakis LJ, Moskowitz CH. The role of autologous stem cell transplantation in the treatment of diffuse large B-cell lymphoma in the era of CAR-T cell therapy. Hemasphere. 2019;3(6):e295.

9. Vaughn JE, Anwer F, Deeg HJ. Treatment of refractory ITP and Evans syndrome by haematopoietic cell transplantation: is it indicated, and for whom? Vox Sang. 2016;110(1):5-11.

10. Huhn RD, Fogarty PF, Nakamura R, Read EJ, Leitman SF, Rick ME, Kimball J, et al. High-dose cyclophosphamide with autologous lymphocyte-depleted peripheral blood stem cell (PBSC) support for treatment of refractory chronic autoimmune thrombocytopenia. Blood. 2003;101(1):71-77.

11. Passweg JR, Rabusin M. Hematopoetic stem cell transplantation for immune thrombocytopenia and other refractory autoimmune cytopenias. Autoimmunity. 2008;41(8):660-665. 